\title{
Discovery of a coherent 87.65-minute oscillation of the suspected cataclysmic variable NSV 2872 (Aur 2) ${ }^{\star}$
}

\author{
V. P. Kozhevnikov ${ }^{\star}$ \\ Astronomical Observatory, Ural State University, Lenin Av. 51, Ekaterinburg, 620083, Russia
}

Received 16 July 2002 / Accepted 7 November 2002

\begin{abstract}
We report discovery of a low-amplitude optical oscillation at a period of $87.65 \mathrm{~min}$ of the suspected cataclysmic variable NSV 2872. During a month of our observations the oscillation was strictly periodic while the amplitude appeared highly variable. Recently Liu \& $\mathrm{Hu}$ (2000) revealed that the spectrum of this star is not typical of cataclysmic variables, and it is simply an early-K type star. In this connexion the observed oscillation seems rather puzzling. The period and amplitude of the oscillation are typical of the $\delta$ Sct variables, but both the spectrum and the colour of this star indicate that NSV 2872 is outside the instability strip. Our observations of NSV 2872 reveal sure signs of the flickering which is a hallmark of the cataclysmic variables though the activity of the flickering in this star seems rather low. Also the highly variable amplitude of the observed oscillation is typical of the intermediate polar subclass of cataclysmic variables. If NSV 2872 is considered as a cataclysmic variable, it is unlikely that the 87.65 -min oscillation can be related to the orbital period of the system since cataclysmic variables with $\mathrm{K}$ type secondaries of normal chemical compositions can not have such short orbital periods. Hence, NSV 2872 is probably a new intermediate polar.
\end{abstract}

Key words. stars: individual: NSV 2872 - stars: novae, cataclysmic variables - stars: oscillations

\section{Introduction}

Cataclysmic variables (CVs) are interacting binaries that consist of a white dwarf primary accreting matter from a low mass secondary filling its Roche lobe. The path that the transferred matter takes depends strongly on the magnetic field of the white dwarf. A bright accretion disk forms in non-magnetic systems, while matter swirling along field lines releases energy in their magnetic counterparts. In polars (or AM Her stars), the field is strong enough to lock the white dwarf spin in synchrony with the orbit. In intermediate polars (or DQ Her stars), the white dwarf rotation is not synchronized with the orbit, and an offset between the rotation axis and the magnetic dipole axis of the white dwarf causes oscillations in the X-ray and optical wavelength bands. Since CVs are interacting binary systems, their emission from the X-rays to the infrared is generally totally dominated by the release of the gravitational energy of the accreted matter in a disk and/or an accretion column and not by the emission of the stellar components. In most cases CVs show emission line spectra. One of the most striking photometric characteristics common to all $\mathrm{CVs}$ are the variations with amplitudes from some hundredth of a magnitude up to one magnitude which have time-scales ranging from seconds to a few dozen minutes. This phenomenon is called flickering.

\footnotetext{
* Research supported in part by the scientific program "Russian Universities" under grant No. UR.02.01.010.

$\star \star$ e-mail: valerij.kozhevnikov@usu.ru
}

Reviews of CVs are given by Patterson (1984) and Warner (1995).

Since the publications of the CVs catalogue of Downes \& Shara (1993) and its second edition (Downes et al. 1997), several observers (Downes et al. 1995; Liu \& Hu 2000; Liu et al. 1999a, 1999b; Munari \& Zwitter 1998; Munari et al. 1997; Zwitter \& Munari 1994, 1995, 1996) have obtained spectroscopic observations of the unstudied stars included in these catalogues to confirm (or disprove) their status as CVs. However, some CVs have optically tick accretion disks and show weak or no emission lines (at last at low spectral resolution) and may be classified incorrectly (Ferguson et al. 1984). In this we made sure themselves by observing the flickering in FY Per. This observation clearly revealed the CV nature of FY Per whereas Downes et al. (1995) reckoned this star among the Ae-Be Herbig stars because of the absence of pronounced emission lines and the presence of a dark nebula in the neighborhood of this star.

Several unstudied CVs from the catalogue of Downes et al. (1997) were included in our program of high-speed photometry of CVs by means of a multichannel photometer. Our aim was to detect the flickering as a key factor certifying these stars as CVs and then to make extended photometric observations and to search for periodic oscillations. One of the program stars was NSV 2872 (Aur 2). This star was discovered as a variable by Rugemer (1933), and its variability was confirmed by Zinner (1932) and Florja \& Kukarkin (1935). In the NSV catalogue 


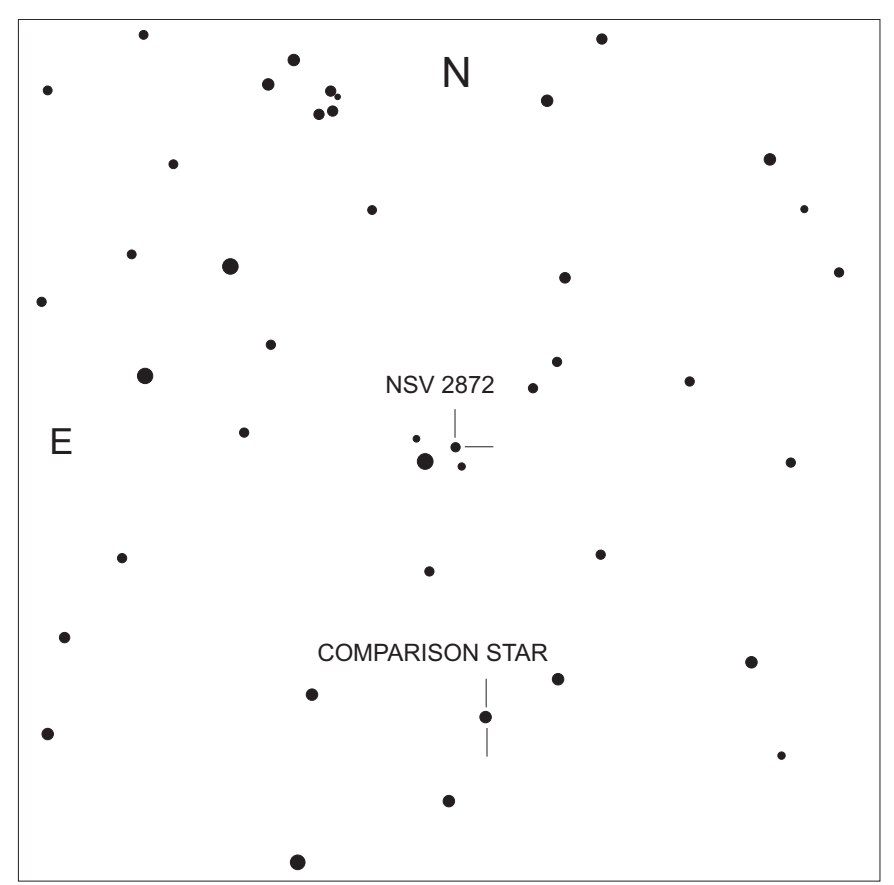

Fig. 1. Field of NSV 2872 with the comparison star used. The side length of the field is 15 arcmin.

(Kholopov et al. 1982) this star is listed as a suspected novalike or U Gem variable with a magnitude range 11.2-14.5. However, on the basis of spectroscopic observations Liu \& $\mathrm{Hu}$ (2000) have concluded that NSV 2872 is not a CV. Despite this fact, we began to observe NSV 2872 in December of 2001, and the first observational night revealed an oscillation at a period of about $1.5 \mathrm{~h}$. The oscillation was clearly visible directly in the lightcurve. The observations were immediately continued in order to define the stability of the period and to establish the coherence of the oscillation. In this paper we present results from all our observations spanning a total duration of 79 hours.

\section{Observations}

NSV 2872 was observed in December of 2001 and in January of 2002 within 11 nights, using a three-channel (two stars+sky) photometer attached to the $70-\mathrm{cm}$ telescope at Kourovka observatory of the Ural State University. A journal of the observations is given in Table 1 . The program and nearby comparison stars were observed through $16^{\prime \prime}$ diaphragms in the first and second channels, and the sky background was observed in the third channel. Figure 1 shows the field of NSV 2872 with the comparison star used. Data were collected at 8-s sampling times in white light (approximately 3000-8000 $\AA$ ), employing a PC based data acquisition system. The design of the photometer is described by Kozhevnikov \& Zakharova (2000). We recently incorporated a CCD guiding system into the photometer. This guiding system enables precise centering of the two stars in the diaphragms to be maintained automatically during an observational night and facilitates the acquisition of long lightcurves which can reach a duration of 11 hours in long winter nights.
Table 1. Journal of the observations.

\begin{tabular}{ccc}
\hline \hline $\begin{array}{c}\text { date } \\
(\mathrm{UT})\end{array}$ & $\begin{array}{c}\text { HJD start } \\
(-2452 \text { 200) }\end{array}$ & $\begin{array}{c}\text { length } \\
\text { (hours) }\end{array}$ \\
\hline 2001 Dec. 11 & 55.21748 & 9.46 \\
2001 Dec. 12 & 56.29948 & 6.58 \\
2001 Dec. 13 & 57.18011 & 10.24 \\
2001 Dec. 15 & 59.15567 & 10.81 \\
2001 Dec. 20 & 64.19519 & 7.40 \\
2001 Dec. 21 & 65.14393 & 4.31 \\
2002 Jan. 3 & 78.09462 & 4.55 \\
2002 Jan. 7 & 82.14418 & 6.43 \\
2002 Jan. 9 & 84.21762 & 6.18 \\
2002 Jan. 10 & 85.41780 & 4.76 \\
2002 Jan. 16 & 91.15610 & 8.12 \\
\hline
\end{tabular}

The continuous measurements of the sky background were subtracted from the program and comparison star data, taking into account the differences in light sensitivity between the channels. Then we took differences of magnitudes of the program and comparison stars. Because the angular separation between the program star and comparison star is only a few arcminutes, the differential magnitudes are corrected for first order atmospheric extinction and light absorption by thin clouds appeared sometimes during the observations.

The mean counts are 17100, 25100,4700 and 4400 for NSV 2872, the comparison star and the sky background in the first and second channels, accordingly. These counts result in the photon noise (rms) of the differential lightcurves, which is equal to $14 \mathrm{mmag}$. The actual rms noise of the differential lightcurves includes also the noises due to atmospheric scintillations and motion of the star images in the diaphragms. We estimate the scintillation noise roughly equal to $4 \mathrm{mmag}$. Unfortunately, the noise caused by motion of the star images was variable. Due to low altitudes above the horizon the scatter of points in some parts of the long lightcurves was found slightly larger than in the best conditions near the zenith. We estimate this noise approximately equal to the photon noise in these cases and negligible near the zenith. The total rms noise of the lightcurves is equal to 15-20 mmag. Figure 2 presents the most long differential lightcurves of NSV 2872 with magnitudes averaged over 160-s time intervals. The rms noise of these lightcurves is smaller by a factor of $\sqrt{20}$ and equal to 3-4 mmag. Besides the white noise components every photometric system usually shows the $1 / f$ noise component which decreases the precision at frequencies approximately below $1 \mathrm{mHz}$ (e.g., Young et al. 1991). This noise component in our photometer equals approximately $1 \mathrm{mmag}$. A detailed description of the noise analysis carried out for the photometer is given by Kozhevnikov \& Zakharova (2000).

\section{Analysis and results}

As seen in Fig. 2, most of the lightcurves clearly show the periodic oscillation. Low-frequency trends which are apparently caused by differential extinction are also visible in the lightcurves. These trends were removed by subtraction of a 2nd order polynomial fit to each lightcurve. Besides, in order 


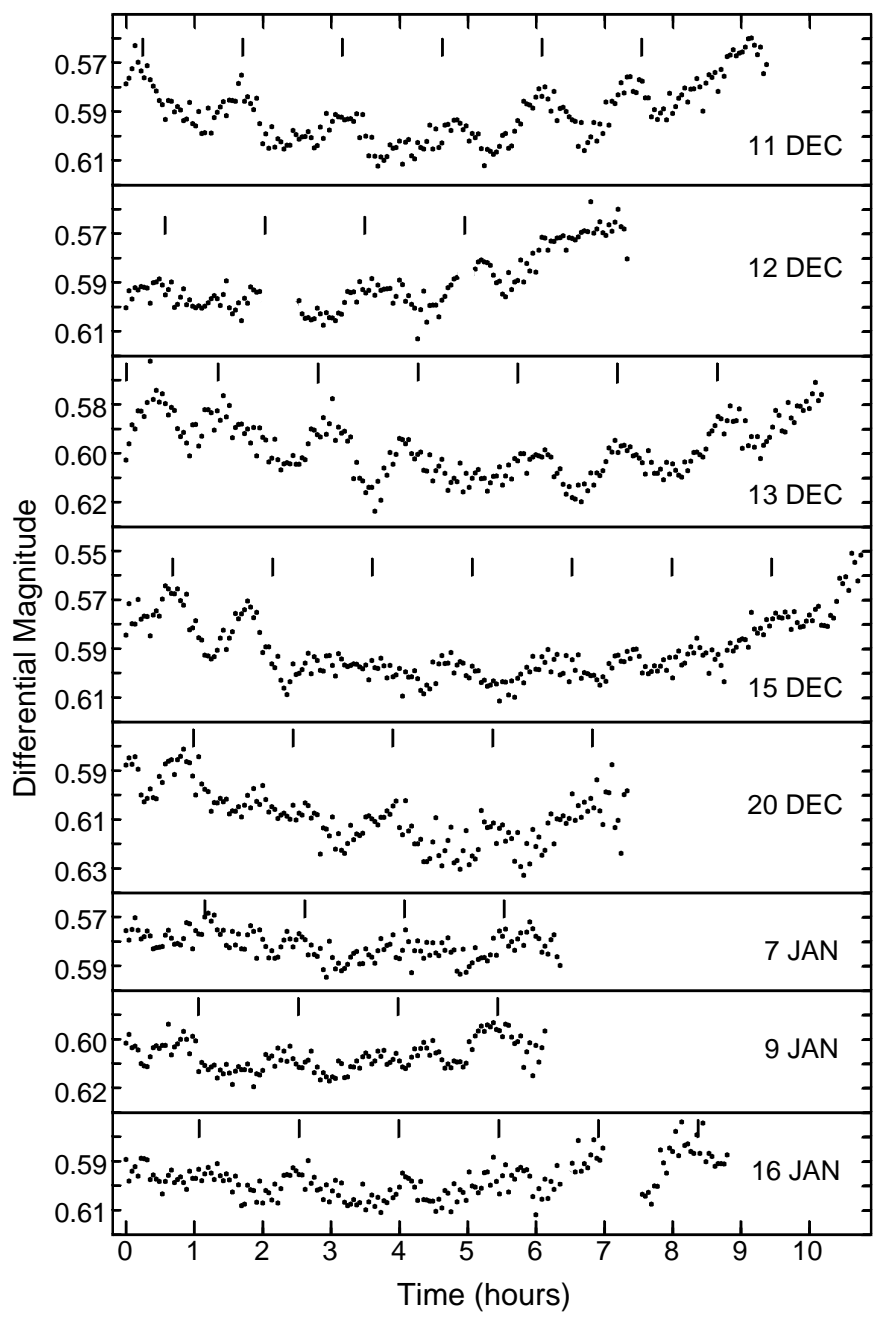

Fig. 2. Differential lightcurves of NSV 2872. The magnitudes are averaged over 160-s time intervals. The rms noise of the lightcurves equals 3-4 mmag. Marks indicate the positions of the maxima of the lightcurves according to a linear ephemeris (see text below).

to prevent distortion of amplitude spectra caused by discontinuity of the data, the lightcurves were smoothed by multiplication of a half-wave of a sinusoid and $10 \%$ of the data at the beginning of the lightcurves and at the end of the lightcurves. Then we calculated the amplitude spectrum for each long lightcurve, using a fast Fourier transform (FFT) algorithm. Our longest lightcurve consists of 243 points (160-s time resolution). Since the FFT algorithm can operate only if lightcurves have a length of a power of 2 , the lightcurves were padded with zeroes up to 256 points. The corresponding amplitude spectra consist of 128 frequency components with frequency bins of $0.024414 \mathrm{mHz}$. These amplitude spectra are shown in Fig. 3. Half of them (December 11, 13 and 20 and January 16) display the prominent peaks at a frequency of $(0.195 \pm 0.012) \mathrm{mHz}$. We consider the error equal to half the frequency bin. This frequency corresponds to a period of $(86 \pm 6) \mathrm{min}$. The other amplitude spectra (Fig. 3) also reveal the peaks at this frequency but these peaks do not exceed the nearby "noise" peaks.

In order to evaluate the statistical significance of detection of the observed oscillation, we calculated an average

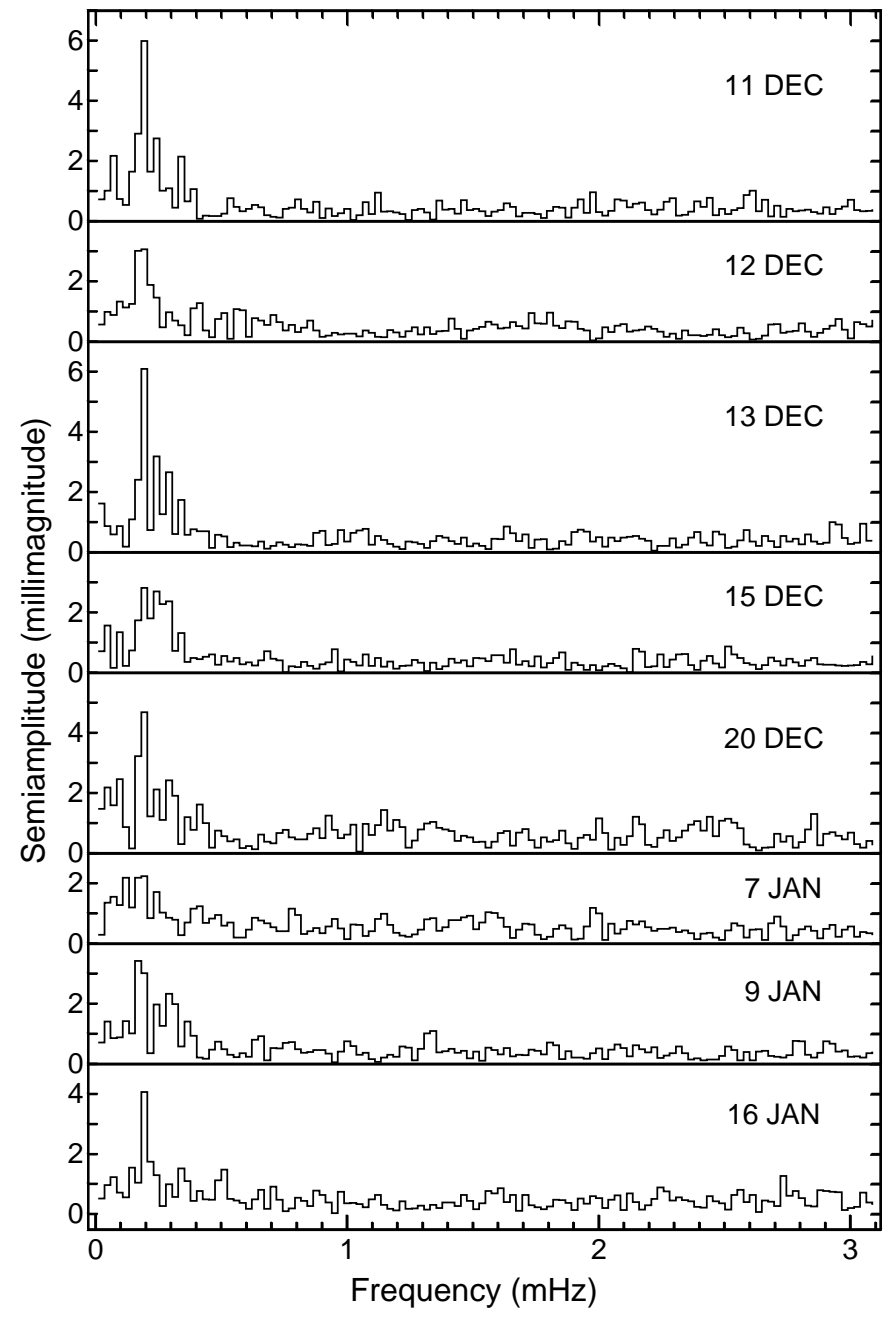

Fig. 3. Amplitude spectra of NSV 2872. Most of them show prominent peaks at a frequency of $(0.195 \pm 0.012) \mathrm{mHz}$. This frequency corresponds to a period of $(86 \pm 6) \mathrm{min}$.

power spectrum. The amplitude spectra were divided by $\sqrt{2}$ and squared. Then we took an average of these squared amplitude spectra. The average power spectrum is given in Fig. 4. The large peak at a frequency of $0.195 \mathrm{mHz}$ is easily visible in this power spectrum. Taking into account that each frequency component in an average power spectrum is distributed as $\chi^{2}(v) / v$, where $v$ is the number of degrees of freedom (e.g., Bendat \& Piersol 1986), we can find the probability that this peak is a chance noise fluctuation. In our case the number of degrees of freedom, $v$, equals 16 because of averaging of eight power spectra. For the large peak at a frequency of $0.195 \mathrm{mHz}$, this probability is much less than $0.05 \%$. Obviously, the oscillation at a period of $(86 \pm 6) \mathrm{min}$ is real beyond all manner of doubt.

Both the lightcurves (Fig. 2) and the amplitude spectra (Fig. 3) show that the amplitude of the oscillation is highly variable. The oscillation semiamplitude varies approximately in range 3-8 mmag from night to night as well as during some nights. The striking example of this variability is revealed in the lightcurve obtained on December 15 when the oscillation semiamplitude faded from approximately $8 \mathrm{mmag}$ at the beginning 


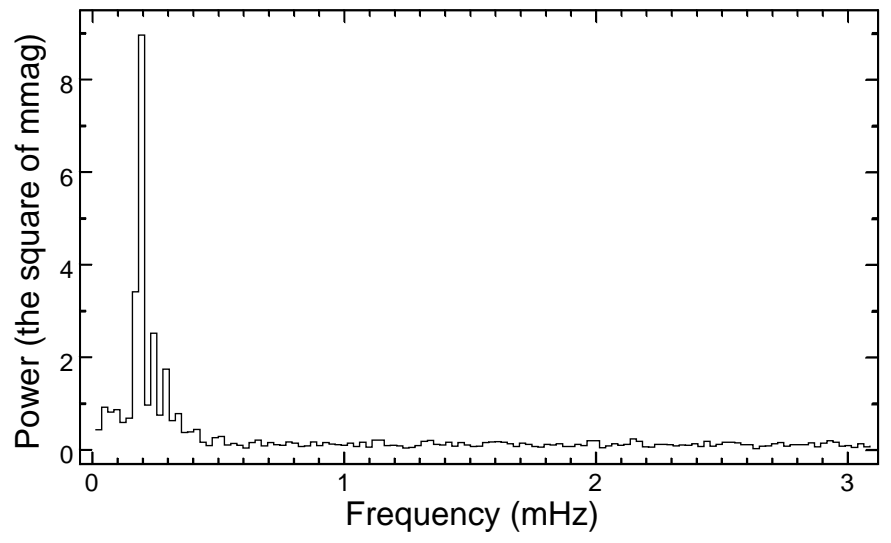

Fig. 4. Average power spectrum of NSV 2872. The large peak at a frequency of $(0.195 \pm 0.012) \mathrm{mHz}$ reveals the oscillation at a period of $(86 \pm 6) \mathrm{min}$. The increased noise level at frequencies in range $0.2-0.4 \mathrm{mHz}$ point to the flickering though its activity seems rather low.

of the night up to the nearly complete disappearance of the oscillation at the end of the night.

Some periodic oscillations can be caused by atmospheric and instrumental effects. If any observed oscillation is phase coherent during all the observational nights, it can not be caused by such reasons because the coherence in itself is the best filter which eliminates most of the artificial signals. The coherence of the observed oscillation will be analysed below. Nevertheless, it is worth while to show the opportune example of an observation of another suspected CV, namely NSV 1725 (Per 1) which does not reveal any oscillations. This is also useful in order to define the noise of the photometer. We observed this star on December 8 just before the observations of NSV 2872, using the same observational technique and the same data processing. Corresponding to this observation, the lightcurve and the amplitude spectrum are shown in Fig. 5. The rms noise is calculated directly from the lightcurve after the removal the low frequency trends and equals 4 mmag. The amplitude spectrum (the lower frame of Fig. 5) does not reveal any significant peak which might indicate an oscillation. The $1 / f$ noise level at frequencies in range $0.2-0.4 \mathrm{mHz}$ in this spectrum does not exceed 1 mmag and only slightly exceeds the white noise level at higher frequencies.

Most of the CVs show the flickering which is visible directly in the lightcurves (e.g., Bruch 1992). In this respect the lightcurves of NSV 2872 (Fig. 2) are not typical of CVs though the shapes of some oscillation cycles seem distorted and may indicate that the star perceptibly flickers. The careful inspection of the amplitude spectra (Fig. 3) reveals that some of them (December 13 and 15 and January 9) have the increased noise levels at frequencies in range $0.2-0.4 \mathrm{mHz}$ (exactly behind the peaks indicating the oscillation). These noise levels are significantly higher than the noise levels at higher frequencies and reach 2-3 mmag. They are significantly higher than the noise level at the corresponding frequencies in the amplitude spectrum of NSV 1725 (Fig. 5). Also the noise level at frequencies in range $0.2-0.4 \mathrm{mHz}$ in the average power spectrum (Fig. 4) is higher than the noise levels at the same frequencies in the
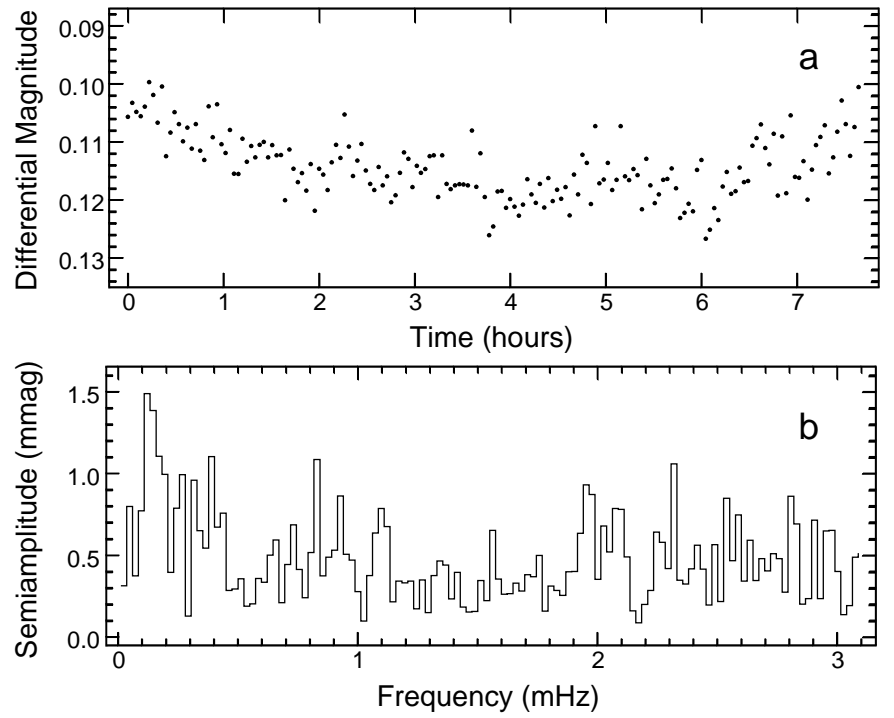

Fig. 5. Lightcurve (the frame a)) and amplitude spectrum (the frame b)) of the suspected cataclysmic variable NSV 1725. The rms noise of the lightcurve equals $4 \mathrm{mmag}$. The amplitude spectrum does not reveal any periodic oscillations. The $1 / f$ noise level at frequencies in range $0.2-0.4 \mathrm{mHz}$ does not exceed $1 \mathrm{mmag}$.

power spectra which we obtained, carrying out the noise analysis for the photometer (see Fig. 2 of Kozhevnikov \& Zakhariva 2000). Obviously, these increased noise levels in the amplitude and power spectra are caused by the flickering in NSV 2872. Thus, both the lightcurves and the spectra of NSV 2872 reveal the flickering though its activity seems considerably weaker than in other CVs.

In order to evaluate more accurately the period of the observed oscillation and to define its stability we calculated power spectra, using all the lightcurves together and the lightcurves obtained in December and in January separately. For this purpose we constructed three time series consisting of all our lightcurves including the short observations. The magnitudes were averaged over 160-s time intervals for the data sets obtained in December and in January and over 400-s time intervals for all our data sets together. All the gaps caused by the absence of observational data were padded with zeroes. In addition, these three time series consisting of 5458, 7253 and 7843 points were padded with zeroes up to 8192 points, and the power spectra were calculated using the FFT algorithm. Each of these power spectra consists of 4096 frequency components with frequency bins of $0.000762 \mathrm{mHz}$ for the data sets obtained in December and in January and $0.000305 \mathrm{mHz}$ for all the data sets together. Low-frequency regions of these power spectra are presented in Fig. 6. Two highest peaks in the power spectra of the data sets obtained in December and in January appeared strictly at the same frequency which equals $(0.18974 \pm 0.00038) \mathrm{mHz}$. This means that within the accuracy provided with these power spectra the oscillation period is stable and equals $(87.84 \pm 0.18) \mathrm{min}$. As before, we consider the error equal to half the frequency bin. Using all the data sets, we can evaluate the oscillation period more accurately. The highest peak in the corresponding power spectrum (the lower frame of Fig. 6) appeared at a frequency of $(0.19002 \pm 0.00015) \mathrm{mHz}$. 


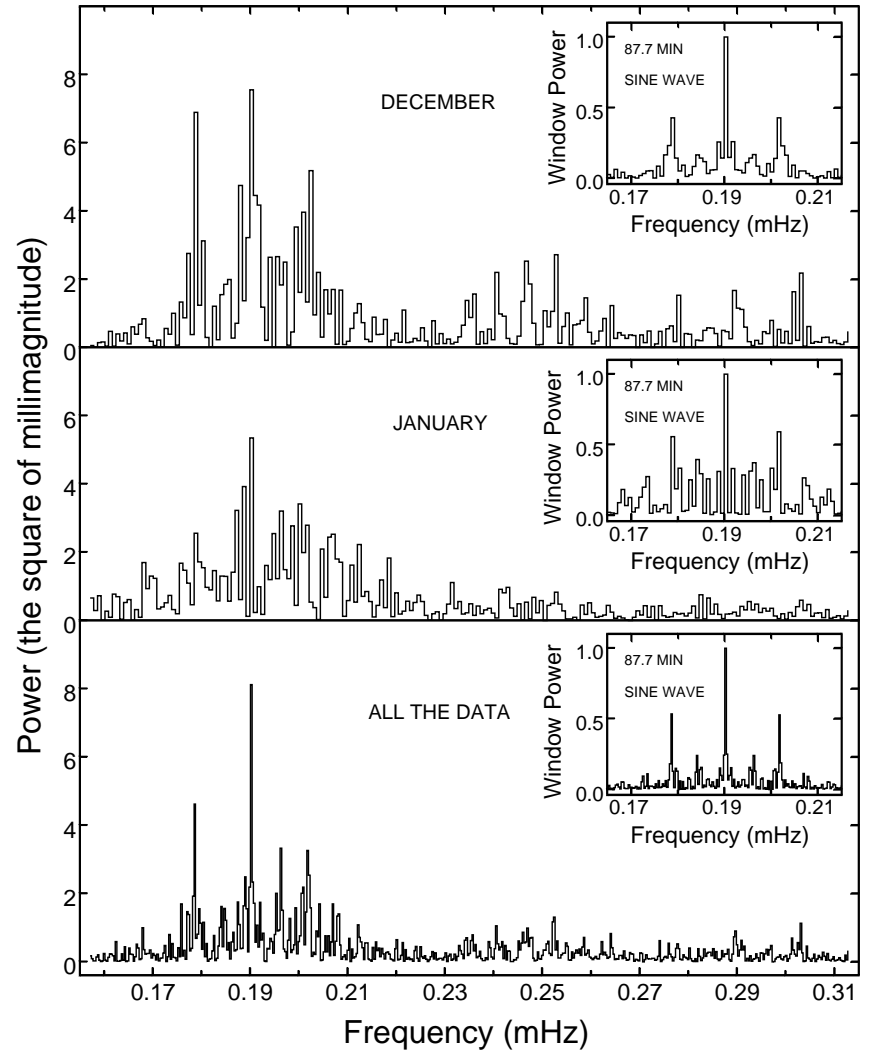

Fig. 6. Power spectra of NSV 2872 that are calculated using the data sets obtained in December, in January and all the data sets together. Two largest peaks in the upper and middle frames strictly coincide in frequency. The largest peak in the lower frame is agreed in frequency with the former two peaks and has a lesser width due to a larger observational coverage. The inserted frames show the window functions obtained using a sine wave with the gaps simulating the absence of observational data.

This frequency corresponds to a period of $(87.71 \pm 0.07) \mathrm{min}$ and quite agrees with the results obtained using the data sets of December and January.

In order to find out the structures of the window functions, we constructed three time series consisting of a sine wave having a period of $87.7 \mathrm{~min}$ with the gaps simulating the absence of observational data, according to the time of our observations. These window functions are inserted into Fig. 6. Obviously, the power spectra of the data of December and January are rather noisy and resemble the window functions only slightly. Nevertheless, they clearly show the large peaks corresponding to the oscillation at a period of $87.7 \mathrm{~min}$.

One more method may be used in order to evaluate more accurately the oscillation period and to examine, whether other periodic oscillations (besides aliases) are hidden in noise or not. This is classical one-way analysis of variance (AoV) (e.g., Afifi $\&$ Azen 1979) in application to data folded and grouped into bins according to the phase of a trial period (SchwarzenbergSzerny 1989). Since the test statistic $\Theta_{\mathrm{AoV}}$ has $F$ distribution with $r-1$ and $n-r$ degrees of freedom, where $r$ is the number of bins and $n$ is the total number of observations, the statistical significance of detection of periodic signals can be estimated. We tried AoV periodograms in search of periodic

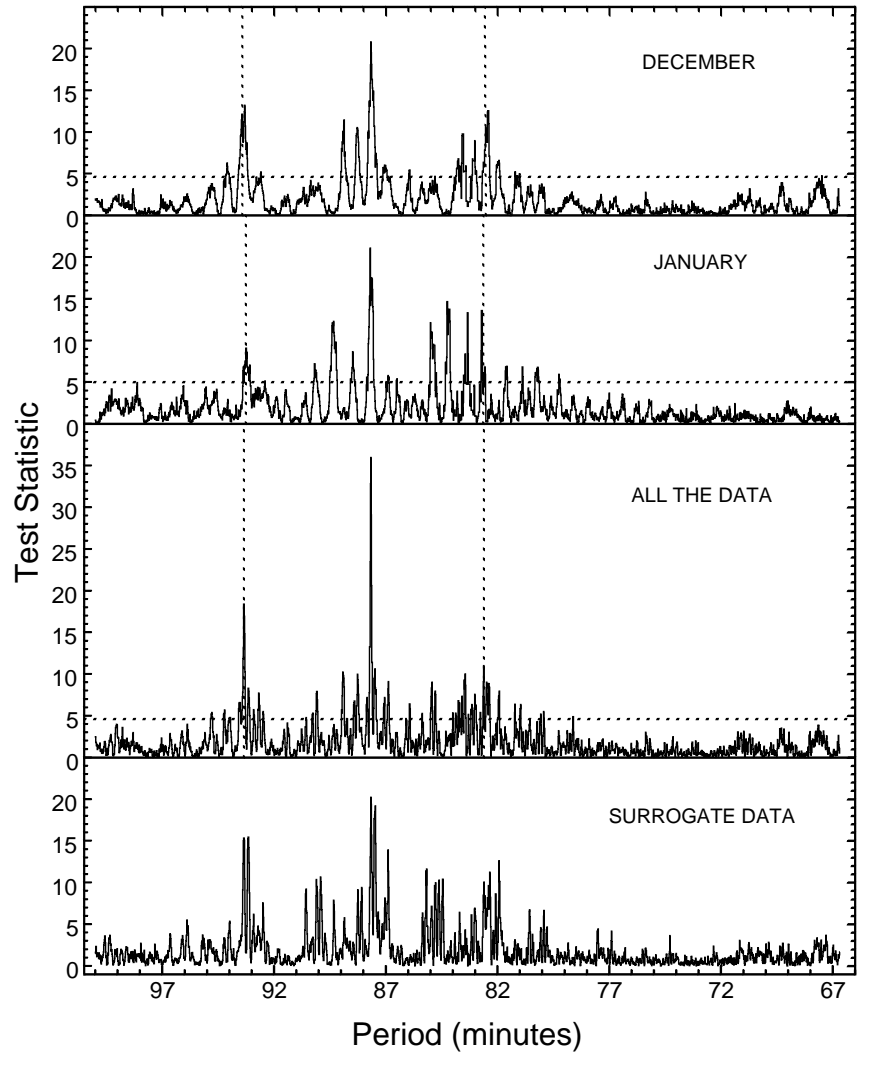

Fig. 7. AoV periodograms of NSV 2872. Each periodogram consists of 2000 points. The steps of change in trial periods equal $1 \mathrm{~s}$. The horizontal dotted lines indicate the $0.1 \%$ significance level. The vertical dotted lines mark the positions of the one-day aliases. The periodogram of the surrogate data is calculated by changing the time of the beginning of the observations of each night randomly with a standard deviation of $10 \%$ of the observed period. A large amount of the chaotically situated significant lines shows that such a procedure entirely destroys the structure of the AoV periodogram.

oscillations of the intermediate polar V709 Cas and found their better statistical properties in comparison with power spectra to detect weak periodic signals (Kozhevnikov 2001). In addition, AoV periodograms seem useful in order to establish the coherence of the observed oscillation.

The AoV periodograms of NSV 2872 are shown in Fig. 7. We used 5 bins per phase for each trial period. The magnitudes were previously averaged over 1000 -s time intervals. The numbers of the observations are 175 in December and 109 in January. The critical value of the test statistic $\Theta_{\mathrm{AoV}}$ for the $0.1 \%$ significance level, which is shown in Fig. 7 as horizontal dashed lines, corresponds to $r-1=4$ and $n-r>120$ and equals 4.62 in the upper and lower frames. The $0.1 \%$ significance level in the middle frame is somewhat higher due to the smaller number of observations and is approximately equal to 5.0 .

Three highest lines in the AoV periodograms (Fig. 7) reveal the periods which equal $(87.63 \pm 0.10) \mathrm{min}$ in December, $(87.65 \pm 0.10) \mathrm{min}$ in January and $(87.65 \pm 0.03)$ min when we used all the data sets together. We consider the error equal to half the line width between half intensity points. All the periods are entirely agreed with each other and indicate that 
the observed oscillation has the stable period. The vertical dotted lines mark the positions of the one-day aliases that are obtained from AoV periodograms of a sine wave having a period of $87.65 \mathrm{~min}$ with the gaps simulating the absence of observational data. As seen in Fig. 7, the one-day aliases have the slightly different positions in December and in January that are apparently caused by particularities in the time of the observations. The coincidence of the real one-day aliases and their calculated positions obtained from a sine wave with the gaps simulating the absence of observational data is also evidence that the observed oscillation has the stable period.

After the period of the oscillation was precisely evaluated we attempted to place a comb of marks indicating the maxima of the oscillation in Fig. 2, according to a linear ephemeris. The following linear ephemeris gives satisfactory results:

$T_{\max }(\mathrm{HJD})=2452255.2277( \pm 10)+0.060868( \pm 5) E$.

As seen in Fig. 2, in many cases these marks precisely coincide with the ridges of the pronounced maxima. The ridges of some other maxima seem to deviate only slightly, and these deviations are both positive and negative. In several cases the maxima seem to be absent. This is quite natural in the presence of the noise caused by the flickering. Only two pronounced maxima deviate significantly from the ephemeris. The significant deviation of the second maximum of the lightcurve obtained on December 15 can be explained by the fading of the oscillation on this night, which occured exactly after this maximum (as seen in Fig. 2). Probably, the significant deviation of the first maximum in the lightcurve obtained on December 13 may also be explained in such a way. Unfortunately, we do not know the oscillation behaviour before this maximum.

Such a simple comparison of the positions of the maxima of the lightcurves and the marks according to the ephemeris can give only a superficial comprehension of the coherence of the observed oscillation in the presence of the noise caused by the flickering. As a rule, periodic oscillations that are observed in some other CVs are completely hidden in noise, and their coherence may be estimated using only power spectra and/or periodograms. Theoretically the frequency composition of a strictly periodic signal is a delta-function, and the width of the corresponding peak in a power spectrum must be determined by the frequency resolution which improves with the extension of the observations. In our case the situation is somewhat complicated due to the gaps in the data, and each peak corresponding to a strictly periodic signal must be a window function. While the noise impedes to see the clear structure of the window functions in the data obtained in December and in January, the power spectra of NSV 2872 (Fig. 6) show that in all the cases the maximum power of the observed oscillation is concentrated in the only frequency component, and its width simply equals to the frequency resolution of the corresponding spectrum. This is the very thing that is expected in the case of the strictly periodic oscillation. Also the relative height of the principal peaks grows with the improving of the frequency resolution and with the extension of the observations. This is the very property which allows hidden periodic signals to be detected (e.g., Bendat \& Piersol 1986). Thus, the power spectra of NSV 2872 do not show any signs of the poor coherence of the oscillation.

The principal lines in the AoV periodograms (Fig. 7) show the same behaviour with the extension of the observations as in the power spectra, namely the width decreasing and the height increasing. Obviously, it is also the sure signs that the observed oscillation is strictly periodic. In addition, employing small steps in change of trial periods, we can analyse the shape of the principal line and its surrounding in detail and compare it with the line of an obviously periodic artificial signal, e.g., a sine wave. In Fig. 8 we show an expanded view of the periodogram obtained from all the data sets together and the periodogram obtained from a sine wave having a period of $87.65 \mathrm{~min}$ with the gaps simulating the absence of observational data. In order to obtain the more realistic periodogram of the sine wave, the artificial white noise was added previously to this sine wave. As seen in Fig. 8, the shapes of the principal lines in these periodograms coincide. This means that the frequency composition of the observed oscillation is the same as in the case of the strictly periodic signal in the presence of the noise. Moreover, one can easily see that the positions of most of the perceptible lines that surround the principal lines coincide also in these periodograms though these lines have different heights due probably to the variable amplitude of the observed oscillation. These lines are actually different aliases caused by particularities in the time of the observations and outline the window function. Such a distinct structure of the AoV periodograms can not be obtained if any of these oscillations is not coherent. In order to demonstrate this, we calculated an AoV periodogram of the surrogate data for NSV 2872 by changing the time of the beginning of the observations of each night randomly with a standard deviation of $10 \%$ of the observed period. As seen in the lower frames of Figs. 7 and 8, even such a small random jumps of the oscillation phase from night to night can completely destroy the structure of the AoV periodogram.

The latter numerical experiment clearly shows that the observed oscillation has a high degree of coherence from night to night. However, it is incorrect to consider that the oscillation might have the poor short-term coherence during each night, and this might cause the excess power in range $0.2-0.4 \mathrm{mHz}$ (Fig. 4). The frequency deviations to the higher frequencies, if will occur, must be accompanied by the frequency deviations to the lower frequencies since otherwise the oscillation frequency will change from night to night too. In such a case the oscillation will have a certain frequency composition and will not show the decreasing of the width of the peaks and lines in the power spectra and periodograms with the improving of the frequency resolution caused by the extension of the observations since their width will be determined by this frequency composition. Thus, we find ample evidence that the observed oscillation is strictly periodic and observed in the presence of the noise caused by the flickering. In Table 2 we give the summary of the evaluations of the periods of the oscillation.

\section{Discussion}

As mentioned, Liu \& Hu (2000) have not found spectroscopic evidence of the CV nature of NSV 2872. Instead, the spectrum 


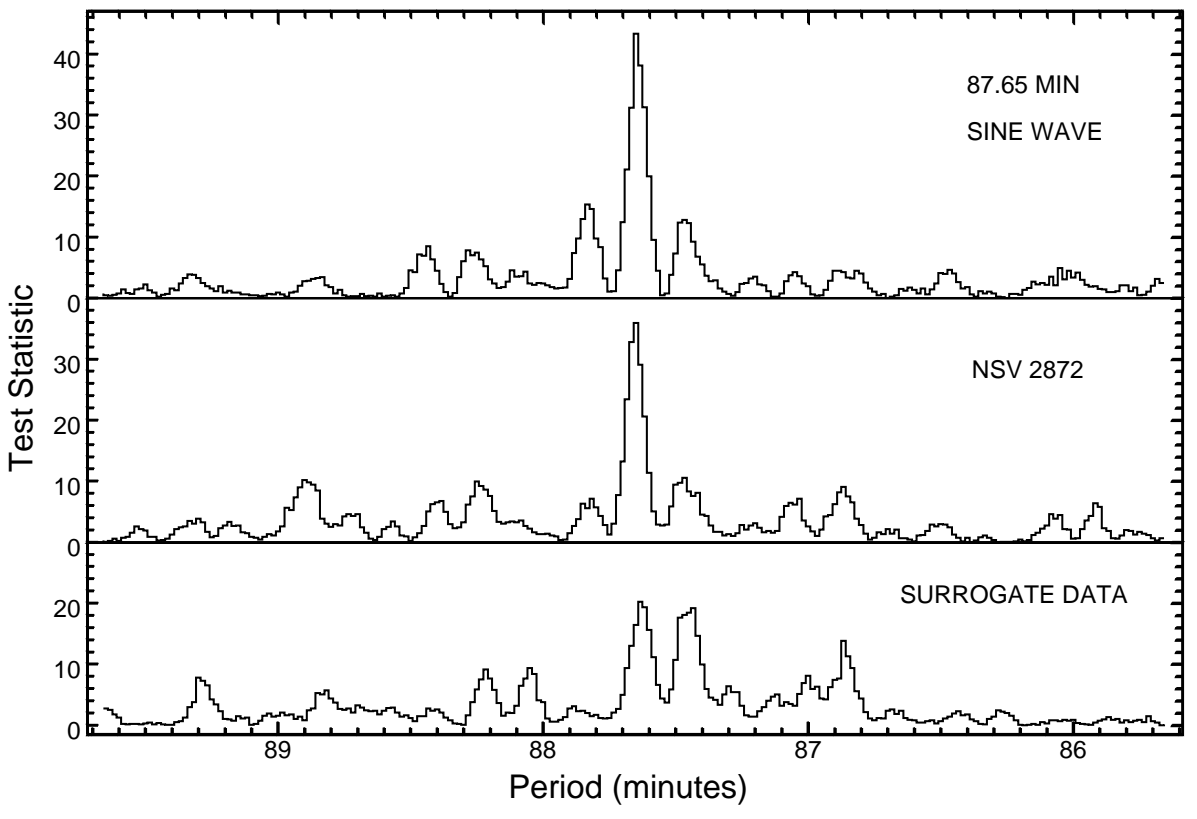

Fig. 8. An expanded view of the AoV periodograms of NSV 2872 (a part of the lower frame of Fig. 7) and a sine wave having a period of $87.65 \mathrm{~min}$ with the gaps simulating the absence of observational data. The shapes of the principal lines coincide in these periodograms. The positions of most of the perceptible lines near the principal lines coincide in these periodograms too. These lines are apparently caused by particularities in the time of the observations and outline the window function. The AoV periodogram of the surrogate data for NSV 2872 shows that even small random jumps of the oscillation phase from night to night can entirely destroy the structure of the AoV periodogram.
Table 2. Summary of the evaluations of the periods.

\begin{tabular}{lcc}
\hline \hline \multirow{2}{*}{ time } & \multicolumn{2}{c}{ period (minutes) } \\
\cline { 2 - 3 } interval & power spectra & AoV periodograms \\
\hline December & $87.84 \pm 0.18$ & $87.63 \pm 0.10$ \\
January & $87.84 \pm 0.18$ & $87.65 \pm 0.10$ \\
all the data & $87.71 \pm 0.07$ & $87.65 \pm 0.03$ \\
\hline
\end{tabular}

obtained by Liu \& Hu (2000) revealed an early-K type star with weak Balmer absorption and other typical features. In this connexion we are in difficult situation in order to interpret the observed oscillation. If NSV 2872 is not a CV, then how we can account for the oscillation at a period of $87.65 \mathrm{~min}$ ?

The period and the amplitude of the observed oscillation suggest that NSV 2872 may be a $\delta$ Sct variable. However, both the spectrum and the $(B-V)$ colour index that equals $1.1 \mathrm{mag}$ (Liu \& $\mathrm{Hu} 2000$ ) show that this star is outside the instability strip (see, e.g., Rodriguez et al. 1994 about properties of the $\delta$ Sct variables). In some of our observational nights we conducted observations of NSV 2872 and the comparison star, using one of the channels of our photometer as a simple one-channel photometer and two standard filters to obtain $B$ and $V$ magnitudes. We used the magnitudes of two standard stars in the vicinity of SS Aur (Khruzina \& Shugarov $1991)$ which is situated not far from NSV 2872. The $(B-V)$ colour indexes and their standard deviations are found equal to $(1.14 \pm 0.02) \mathrm{mag}$ and $(0.39 \pm 0.01) \mathrm{mag}$ for NSV 2872 and the comparison star, accordingly. These measurements show that NSV 2872 is really a red star and can not be a $\delta$ Sct variable.

If the colour of NSV 2872 is incompatible with the colours of the $\delta$ Sct variables, then the comparison star might be a $\delta$ Sct variable. After all, its colour is typical of the $\delta$ Sct variables. However, it proves to be not a simple problem to decide which of the two stars is really a variable since the atmospheric transparency fluctuations are comparable with the amplitude of the observed oscillation, and the oscillation is directly visible only in the differential lightcurves.

In order to solve this problem, we used technique based on Fourier transforms. In the observation obtained on December 11 when the 87.65 -min oscillation was best visible (see Fig. 2) we chose three segments of data of NSV 2872, the comparison star and the differential lightcurve. These data segments correspond to the best transparency conditions on this night and are strictly four oscillation cycles in length. Using a discrete Fourier transform algorithm, we calculated the Fourier transforms of these segments, and, removing all the frequency components except the fourth ones, we reconstructed the filtered lightcurves by means of an inverse Fourier transform algorithm. These filtered lightcurves are shown in Fig. 9. It is not surprising that the filtered lightcurve of the comparison star (Fig. 9a) is a sine wave. Since a Fourier transform is simply a set of different sine waves, then, removing all the sine waves besides the only sine wave from the Fourier transform of the segment of the comparison star data, we have obtained the noise component of such a shape. Fortunately, in this case the filtered lightcurves of the comparison star (Fig. 9a) and NSV 2872 (Fig. 9b) turn out to have the similar phases, and we can definitely conclude that the 87.65-min oscillation belongs to NSV 2872 because the filtered lightcurve of NSV 2872 has the significantly larger amplitude. The difference of these two filtered lightcurves exactly gives the sine wave identical to the fourth frequency component of the differential lightcurve and entirely agrees with the points of the differential lightcurve (Fig. 9c). Thus, the careful analysis definitely shows that the 87.65-min oscillation was observed in NSV 2872.

Solar type stars with effective temperatures below $7000 \mathrm{~K}$ have a convective layer in their outer parts and so might be expected to undergo oscillations in the high-frequency domain. However, solar type stars oscillations must have very low amplitudes of a few ppm, and their detection remain impossible, using photometry from the ground. This is a challenging 


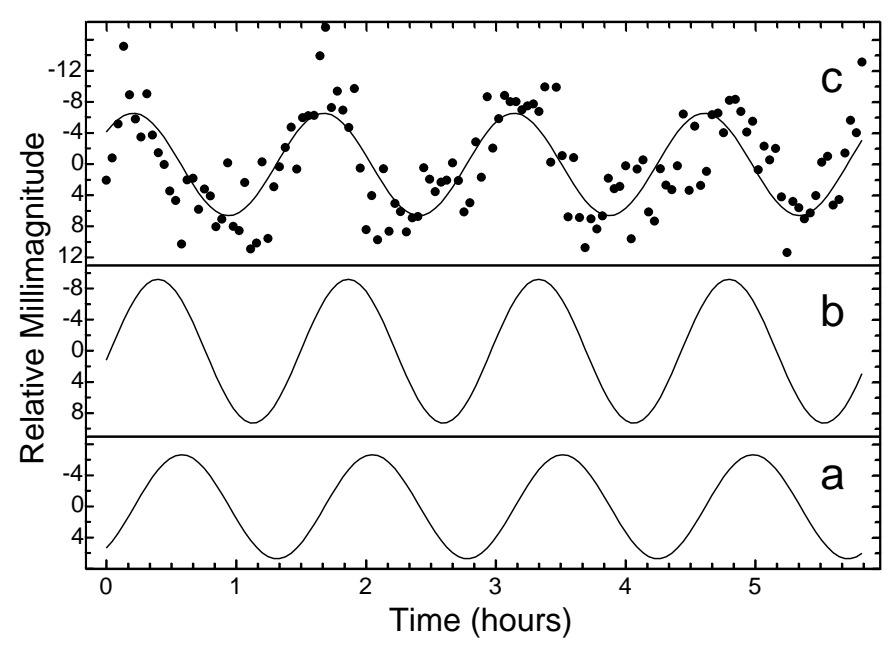

Fig. 9. Sine waves corresponding to the fourth frequency components. They are calculated using Fourier transforms from the data segments obtained on December 11 and are shown for the comparison star in the frame a) and for NSV 2872 in the frame b). These sine waves can not agree with the observational points of the corresponding lightcurves since the lightcurves are distorted by the atmospheric noise at other frequencies. However, their difference (the frame c) perfectly agrees with the points of the differential lightcurve. The significantly greater amplitude of the sine wave in the frame b) demonstrates that the 87.65 -min oscillation is the property of NSV 2872 but not of the comparison star.

problem of asteroseismology (e.g., Kjeldsen \& Bedding 1995). If NSV 2872 is a K type solitary star, we can not account for the 87.65 -min oscillation in such a way because the expected oscillation amplitude is much below the detection threshold. Thus, we have no choice but to consider the possibility that NSV 2872 is related to CVs, since short-period oscillations are typical of such stars.

First of all, if the secondary in this conjectural binary system is a $\mathrm{K}$ type star, it excludes the possibility that the 87.65-min oscillation might be related to the orbital period. CVs with $\mathrm{K}$ type secondaries of normal chemical compositions possess the great angular momentum, and the orbital periods of such systems must be above $5 \mathrm{~h}$ (see, e.g., Fig. 5 of Beuermann et al. 1998). The only known dwarf nova 1RXS J232953.9+062814 with an orbital period of $64 \mathrm{~min}$ have a $\mathrm{K}$ type secondary. It is considered, that the secondary in this system has undergone substantial nuclear evolution, leading to an enhanced helium abundance (Thorstensen et al. 2002). We believe that such an exotic case can be excluded for the NSV 2872 because the lightcurve of 1RXS J232953.9+062814 reveals the double peaked modulation which is consistent with ellipsoidal variations (see Fig. 2 of Thorstensen et al. 2002) whereas the $87.65-\mathrm{min}$ oscillation of NSV 2872 has the highly variable amplitude.

Other possibility that the 87.65 -min oscillation might be the coherent pulsation of the white dwarf primary is also incompatible with the highly variable amplitude of the observed oscillation. Besides, most of the coherent pulsating white dwarfs reveal oscillations in more than one mode and these oscillations have shorter periods typically in range 100-2000 s (e.g., Brown $\&$ Gilliland 1994). Then we have no choice but to attribute the 87.65-min oscillation to the unsynchronously rotating magnetic white dwarf, i.e. NSV 2872 can be an intermediate polar. Besides the stable period, this assumption is also confirmed by the highly variable amplitude of the oscillation. Rapid changes of the oscillation amplitudes are typical of intermediate polars because these oscillations are powered by unstable accretion flows (Patterson 1994).

In order to vindicate the CV nature of NSV 2872, we must account for the absence of emission lines and the presence of absorption lines of a $\mathrm{K}$ type secondary in its optical spectrum. As mentioned, some nova-like variables possess optically thick accretion disks and are characterized by weak or no emission lines in their spectra (at last at low spectral resolution) (Ferguson et al. 1984). However, these are bright systems and their optical emission is completely dominated by the accretion disks (e.g., Patterson 1984; Warner 1987), so the secondaries in such systems can be detected only in the infrared.

On the other hand, a few of the long-period dwarf novae have K type secondaries which contribute considerable parts of optical light in quiescence and can be revealed by their absorption spectra. Examples are DX And (Bruch et al. 1997), GK Per (Crampton et al. 1986), BV Cen (Gilliland 1982), V426 Oph (Hessmann 1988) and RU Peg (Stover 1981). Due to the low accretion luminosity the unusual nova-like variable AE Aqr also displays absorption lines of a $\mathrm{K}$ type secondary (Welsh et al. 1995). But these CVs as well as other quiescent dwarf novae typically show broad intense emission lines (e.g., Kiplinger 1979).

Nova-like variables of the VY Scl subtype (or anti-dwarf novae) in low luminosity states attributed to a cessation (or drastic reduction) of mass transfer at intervals of months or years reveal extremely narrow emission lines which may not be observable at low spectral resolution (Robinson et al. 1981; Shafter et al. 1985). However, most of the VY Scl stars have orbital periods between $3 \mathrm{~h}$ and $4 \mathrm{~h}$ (e.g., Ferbunt 1997) and, hence, can not contain K type secondaries. Thus, if NSV 2872 is not an unusual long-period VY Scl star that contains a $\mathrm{K}$ type secondary and is observed in a low state, this star must permanently have a very low mass transfer rate.

Estimates of the mass transfer rates or absolute magnitudes in CVs (e.g., Patterson 1984; Warner 1987) typically show a large degree of scatter when they are plotted against the binary period. Consistency between this fact as well as the coexistence of various $\mathrm{CV}$ subtypes at similar orbital periods and the theory of their secular evolution demands the existence of the strong fluctuations of the "instantaneous" mass transfer rate about the secular mean, and time-scales of such fluctuations are too long to be directly observable (King et al. 1995, 1996). According to calculations of King et al. (1995, 1996), CVs can oscillate between high states and low, almost detached states due to irradiation of the secondary stars by the accreting component. Such high and low states can last for millions of years, and some of the various $\mathrm{CV}$ subtypes can inhabit specific parts of these fluctuation cycles.

Another reason that CVs can undergo prolonged low states follows from the discrepancy between the space density of classical novae deduced from surveys in the solar neighborhood and the observed nova outburst frequency in $\mathbf{M} 31$ 
(Patterson 1984). This discrepancy as well as the unusually faint absolute magnitudes of a few identified very old novae are evidence for the "hibernation model" of classical novae (Shara et al. 1986; Prialnik \& Shara 1986). According to the hibernation model, old novae remain bright and retain high mass transfer due to irradiation of the secondary by the hot white dwarf for a century after eruption. After the white dwarf cools (a few centuries) mass transfer should slow, and virtually stop. The state of low mass transfer may last many centuries or even millennia. Hibernating novae lack the blue colours and emission lines to be easily discoverable. As time passes, however, the loss of angular momentum due to gravitational radiation and/or magnetic braking diminishes the binary separation and establishes again contact to the Roche lobe. Dwarf novae and nova-like variables are only special states in this long cycle of several millennia, being essentially the same stars as classical novae.

Thus, both observational facts and theoretical arguments testify to the existence of CVs at different states of activity, and less active CVs seem more frequent but hardly discoverable. Then NSV 2872 may be such a low active CV near hibernation. This star may resemble V471 Tau which consists of the K2 type red dwarf and the white dwarf and is usually considered as a typical CV progenitor in which the secondary does not reach its Roche lobe (e.g., Patterson 1984). While the signatures of mass transfer in V471 Tau are subtle at best, it reveals the 555-s oscillation which is attributed to the rotating magnetic white dwarf (e.g., Jensen et al. 1986), i.e. V471 Tau is actually an intermediate polar (Patterson 1994). Bruhweiler \& Sion (1986) have suggested that V471 Tau may have erupted as a nova many centuries ago. If this suggestion is correct then this detached binary is now in a state of deep hibernation (Shara 1989). Since our observations of NSV 2872 reveal sure signs of the flickering which is a hallmark of mass transfer, we can consider that this star hibernates not so deeply as V471 Tau.

\section{Conclusions}

1. We report discovery of an oscillation at a period of $(87.65 \pm$ 0.03 ) min of the suspected cataclysmic variable NSV 2872;

2. During a month of our observations the oscillation revealed the stable period and was phase coherent;

3. The semiamplitude of this oscillation was observed approximately in range 3-8 mmag, showing rapid changes even during a night;

4. Sure signs of the flickering are found in the lightcurves and amplitude spectra of NSV 2872 though its activity seems considerably weaker than in other CVs;

5. Although spectroscopic evidence indicating the $\mathrm{CV}$ nature of NSV 2872 was not found by Liu \& Hu (2000), the very presence of the rapid periodic oscillation with the highly variable amplitude is a hallmark of CV;

6. Since the spectrum obtained by Liu \& Hu (2000) is evidence that the secondary in this system is an early-K type star, we must attribute the 87.65-min oscillation to the unsynchronously rotating magnetic white dwarf but not to the orbital period, i.e. NSV 2872 is probably a new intermediate polar.

\section{References}

Afifi, A. A., \& Azen, S. P. 1979, Statistical Analysis: a Computer Oriented Approax (Academic Press, New York-San FranciscoLondon)

Bendat, J. S., \& Piersol, A. G. 1986, Random Data Analysis and Measurement Procedures (John Wiley \& Sons, Ins.)

Beuermann, K., Baraffe, I., Kolb, U., \& Weichhold, M. 1998, A\&A, 339,518

Brown, T. M., \& Gilliland, R. L. 1994, ARA\&A, 32, 37

Bruch, A. 1992, A\&A, 266, 237

Bruch, A., Vrielmann, S., Hessman, F. V., Kochsiek, A., \& Schimpke, T. 1997, A\&A, 327, 1107

Bruhweiler, F. C., \& Sion, E. M. 1986, ApJ, 304, L21

Crampton, D., Cowley, A. P., \& Fisher, W. A. 1986, ApJ, 300, 788

Downes, R. A., \& Shara, M. M. 1993, PASP, 105, 127

Downes, R., Hoard, D. W., Szkody, P., \& Wachter, S. 1995, AJ, 110, 1824

Downes, R. A., Weblink, R. F., \& Shara, M. M. 1997, PASP, 109, 345

Ferbunt, F. 1997, MNRAS, 290, L55

Ferguson, D. H., Green, R. F., \& Liebert, J. 1984, ApJ, 287, 320

Florja, N. F., \& Kukarkin, B. V. 1935, Peremennye Zvezdy, 5, 19

Gilliland, R. L. 1982, ApJ, 263, 302

Hessman, F. V. 1988, A\&AS, 72, 515

Jensen, K. A., Swank, J. H., Petre, R., et al. 1986, ApJ, 309, L27

Kholopov, P. N., Kukarkin, B. V., Artiukhina, N. M., et al. 1982, New Catalogue of Suspected Variable Stars (Moscow, Nauka)

Khruzina, T. S., \& Shugarov, S. Yu. 1991, Atlas of cataclysmic variables. U Geminorum stars (Moscow Univ. Press)

King, A. R., Frank, J., Kolb, U., \& Ritter, H. 1995, ApJ, 444, L37

King, A. R., Frank, J., Kolb, U., \& Ritter, H. 1996, ApJ, 467, 761

Kiplinger, A. L. 1979, ApJ, 234, 997

Kjeldsen, H., \& Bedding, T. R. 1995, A\&A, 293, 87

Kozhevnikov, V. P. 2001, A\&A, 366, 891

Kozhevnikov, V. P., \& Zakharova, P. E. 2000, in Proc. Euroconference on Disks, Planetesimals and Planets, ed. F. Garzon, C. Eiroa, D. de Winter, \& T. J. Mahoney, ASP Conf. Ser., 219, 381

Liu, W., \& Hu, J. Y. 2000, ApJS, 128, 387

Liu, W., Hu, J. Y., Zhu, X. H., \& Li, Z.U. 1999, ApJS, 122, 243

Liu, W., Hu, J. Y., Li, Z. U., \& Cao, L. 1999, ApJS, 122, 257

Munari, U., \& Zwitter, T. 1998, A\&AS, 128, 277

Munari, U., Zwitter, T., \& Bragaglia, A. 1997, A\&AS, 122, 495

Patterson, J. 1984, ApJS, 54, 443

Patterson, J. 1994, PASP, 106, 209

Prialnik, D., \& Shara, M. M. 1986, ApJ, 311, 172

Robinson, E. L., Barker, E. S., Cochran, A. L., Cochran, W. D., \& Nather, R. E. 1981, ApJ, 251, 611

Rodriguez, E., Lopez de Coca, P., Rolland, A., Garrido, R., \& Costa, V. 1994, A\&AS, 106, 21

Rugemer, H. 1933, Astron. Nachr., 248, 409

Schwarzenberg-Czerny, A. 1989, MNRAS, 241, 153

Shafter, A. W., Szkody, P., Liebert, J., et al. 1985, ApJ, 290, 707

Shara, M. M. 1989, PASP, 101, 5

Shara, M. M., Livio, M., Moffat, A. F. J., \& Orio, M. 1986, ApJ, 311, 163

Stover, R. J. 1981, ApJ, 249, 673

Thorstensen, J. R., Fenton, W. H., Patterson, J. O., et al. 2002, ApJ, 567, L49

Young, A. T., Genet, R. M., Boud, L. J., et al. 1991, PASP, 103, 221

Warner, B. 1987, MNRAS, 227, 23

Warner, B. 1995, Cataclysmic Variable Stars (Cambridge Univ. Press, Cambridge)

Welsh, W. F., Horne, K., \& Gomer, R. 1995, MNRAS, 275, 649

Zinner, E. 1932, Astron. Nachr., 246, 17

Zwitter, T., \& Munari, U. 1994, A\&AS, 107, 503

Zwitter, T., \& Munari, U. 1995, A\&AS, 114, 575

Zwitter, T., \& Munari, U. 1996, A\&AS, 117, 449 